\title{
Defining Access and the Role of Community Care in the Veterans Health Administration
}

\author{
A. Taylor Kelley, MD, MPH, MSc $c^{1,2,3,4}{ }^{\infty}$, Clinton L. Greenstone, MD ${ }^{1,5,6}$, and Susan R. Kirsh, \\ $\mathrm{MD}, \mathrm{MPH}^{\mathrm{P}}$
}

'Department of Internal Medicine, University of Michigan Medical School, Ann Arbor, MI, USA; ${ }^{2}$ National Clinician Scholars Program, Institute for Healthcare Policy and Innovation, University of Michigan, Ann Arbor, MI, USA; ${ }^{3}$ Center for Clinical Management Research, VA Ann Arbor Healthcare System, Ann Arbor, MI, USA; ${ }^{4}$ University of Utah School of Medicine, Salt Lake City, UT, USA; ${ }^{5}$ VA Ann Arbor Healthcare System, Ann Arbor, MI, USA; ${ }^{6}$ Office of Community Care, Veterans Health Administration, US Department of Veterans Affairs, Washington, DC, USA; ${ }^{7}$ Office of Veterans Access to Care, Veterans Health Administration, US Department of Veterans Affairs, Washington, DC, USA.

$\mathrm{J}$ Gen Intern Med 35(5): 1584-5

DOI: $10.1007 / \mathrm{s} 11606-019-05358-\mathrm{Z}$

(c) Society of General Internal Medicine (This is a U.S. government work and not under copyright protection in the U.S.; foreign copyright protection may apply) 2019

$\mathrm{M}$ ore than five years have passed since the Veterans Choice Program (VCP) was established by the United States Congress in response to public outcry for prolonged wait times reported in the Veterans Health Administration (VA). VCP's purpose was to improve access to care by providing VA-enrolled veterans an option to elect care in non-VA settings if travel distance or wait time was excessive (greater than 40 miles or 30 days, respectively). Although adoption was initially slow, within 4 years, VCP grew to account for approximately 30,000 appointments each day at a total cost approaching \$20 billion.

In June of this year, the VA Maintaining Systems and Strengthening Integrated Outside Networks (VA MISSION) Act consolidated VCP and other community care programs into one unified Veterans Community Care Program (VCCP). The new program uncouples fixed qualification criteria from program eligibility and increases VA provider flexibility in making non-VA referrals. Investment in VCCP will surpass \$21 billion, signaling a commitment to increase and streamline non-VA care provided by the VA.

ButtheVApractice of contractingnon-VAcare is notnew.In 1945 - just 15 years after the VAwas established - qualifying veterans enrolled in the VA were authorized to see non-VA providers in their own communities in an effort to reduce preventable hospitalizations. ${ }^{1}$ This preamble to community care, known as the Hometown Program, established an enduring philosophythatuseofnon-VAhealthcareinfrastructure, where VA resources are relatively scarce, is both practical and effective in improving veteran access to care. The VA has relied on

Received July 5, 2019

Revised July 5, 2019

Accepted September 12, 2019

Published online October 25, 2019
non-VA partnerships to deliver healthcare throughout its history.

VCP and VCCP mark a shift in the role of non-VA care in two important ways. First, appointment wait times are now used as an eligibility standard for non-VA care; and second, determination of need for non-VA care is increasingly placed on veterans rather than the VA. These priorities were reaffirmed in the recently announced access standards for VCCP: a 30-min drive or 20-day wait (60 $\mathrm{min}$ or 28 days for specialty care) - a change that will increase the number of veterans eligible for to choose non-VA care by $40 \%$.

As VCCP expands the reach of community care within the VA, several important questions merit attention: How will the program be evaluated? How will appointment wait times be affected? And how will the program empower veterans to make optimal choices when deciding between VA- and nonVA care? Recognition of current challenges - as well as VAdriven initiatives underway in response-may provide a framework for evaluating and improving veteran access to care and identify opportunities for future study.

First, while the VA has led development of wait time measurement in recent years, reliable assessment of wait times to evaluate VCP and VCCP has proved challenging. With the creation of VCP, the VA became the first major healthcare provider to report wait times publicly. However, as the Government Accountability Office reports, reliable measurement of wait times in referrals to community care has remained elusive, and establishing a reliable performance baseline is critical to develop meaningful programmatic evaluation and reform. ${ }^{2}$ The Office of Veteran Access to Care has since begun "mystery shopper" initiatives within the VA to understand wait times and other aspects of accessing healthcare, providing objective observation of veteran experience. As these initiatives extend across VA and non-VA settings, reliability of wait time measurement will likely improve.

Second, wait times used as a basis for veteran eligibility in VCCP have not been measured in nearby non-VA practices, making comparison problematic and rational choice untenable. Notably, legislation for both VCP and VCCP was passed without any analysis of healthcare market comparisons 
between VA and non-VA wait times. However, a subsequent comparison of wait times for new patient appointments showed VA clinics performed as well as community practices in 2014 , and that since 2014 , community practice wait times remained stagnant while wait times in the VA improved. ${ }^{3}$ The study is limited by dissimilar methods for measurement of wait times between VA and non-VA sites; but its findings, if true, question the utility of VCCP as a means to reduce wait times. Value to specific populations, such as rural veterans or veterans requiring frequent or specialized services (especially services unavailable at nearby VA facilities), would not be precluded. But if non-VA settings perform worse than the VA on wait times and other access measures, shifting more veteran care from the VA into the community may not improve access.

With the rollout of the VA MISSION Act, the Office of Community Care has begun recording wait times in community practices using criteria for publicly reported wait times within the VA. By following a standardized set of criteria, the VA will be able to evaluate the timeliness of appointments among contracted community providers and optimize their networks over time. The VA's mystery shopper initiatives provide a promising approach to ongoing validation of reported wait times in both VA and non-VA settings.

Third, additional choice for veterans will require additional decision support. It is unlikely that all veterans more than 30 $60 \mathrm{~min}$ from VA facilities will benefit from receiving care elsewhere, but which veterans will? The movement of veterans and resources from lower cost VA care to higher cost non-VA care could result in loss of services, additional care fragmentation, and even perhaps VA facility closures, paradoxically reducing veteran access. ${ }^{4}$ Conversely, whether nonVA care will have capacity or capability to meet the unique needs of veterans - especially for mental health - remains uncertain. ${ }^{5}$ The VA has, for decades, aligned services with healthcare needs unique to veterans, veteran trust in the VA remains high, and most eligible for VCP choose to wait for VA care. ${ }^{6}$ Development of enhanced shared decision-making approaches between veterans and their providers will therefore be essential.

Key to that process will be the integration of non-VA data. Using VA and newly available non-VA wait time data sources, the Office of Community Care has created a novel decision support tool (DST) that provides veterans and their providers real-time wait time data to help veterans determine where to seek care. The DST will require evaluation and refinement over time, including integration of the DST into virtual care, such as telehealth, and alternative models of care, such as integrated mental health and primary care services. In addition, research methods that link VA and community data provide another promising approach to understand veteran utilization patterns and distribution of risk across health systems - metrics that determine whether veterans at greatest risk are receiving needed care, and may be a means to track changes in health outcomes over time. ${ }^{7}$ Without good information, veterans will be ill-equipped to make choices about their care that lead to the best outcomes.

Continued development and evaluation of access metrics and veteran decision support will enable evaluation aligned with the VA's priorities: optimizing veteran access without compromising quality of care. This may mean some VCCP resources should be applied in ways different than prescribed in the current law. For example, further evaluation of access and quality may find that strengthening existing programs and services within the VA is more cost-effective than alternative approaches. In this new era of community care, the VA must remain true to its history and unwavering in its commitment to use resources in the best ways possible to promote meaningful access to high-quality healthcare for veterans everywhere.

\begin{abstract}
Acknowledgments: The authors acknowledge Dr. Kameron Matthews, MD, JD, and Ranganath Kathawate for their assistance and input in preparing thismanuscript.
\end{abstract}

Corresponding Author: A. Taylor Kelley, MD, MPH, MSc; University of Utah School of Medicine, 50 North Medical Drive 5R341, Salt Lake City, UT 84132-0001, USA

\section{Compliance with Ethical Standards:}

The views and opinions expressed in this article are those of the authors and do not necessarily reflect the official policy or position of the U.S.Department of Veterans Affairs or the U.S. government.

Conflict of Interest: The authors declare that they do not have a conflict of interest.

\section{REFERENCES}

1. Veterans Health Administration Office of Community Care. Fact Sheet 2001: Overview of Non-VA Medical Care. Accessed at https://www.va.gov/ COMMUNITYCARE/docs/pubfiles/factsheets/FactSheet_20-01.pdf: U.S. Department of Veterans Affairs; 2015.

2. United States Government Accountability Office. VETERANS CHOICE PROGRAM: Improvements Needed to Address Access-Related Challenges as VA Plans Consolidation of its Community Care Programs. GAO, 2018 June 4. Accessed at https://www.gao.gov/products/GAO-18-281.

3. Penn M, Bhatnagar S, Kuy S, Lieberman S, Elnahal S, Clancy C, et al. Comparison of Wait Times for New Patients Between the Private Sector and United States Department of Veterans Affairs Medical Centers. JAMA Netw Open. 2019;2(1):e187096.

4. Gellad WF. The Veterans Choice Act and Dual Health System Use. J Gen Intern Med. 2016;31(2):153-4.

5. Tanielian T, Farmer CM, Burns RM, Duffy EL, Setodji CM. Ready or Not? Assessing the Capacity of New York State Health Care Providers to Meet the Need of Veterans. Rand Corporation, March 1, 2018. Accessed at https://www.rand.org/pubs/research_reports/RR2298.html.

6. Veterans Experience Office. VHA Services Surveys Analysis. In: Personal communication from Carolyn Clancy MD, editor. March 15, 2019.

7. Rosen AK, Wagner TH, Pettey WBP, Shwartz M, Chen $\mathbf{Q}$, Lo J, et al. Differences in Risk Scores of Veterans Receiving Community Care Purchased by the Veterans Health Administration. Health Serv Res. 2018;53 Suppl 3:5438-54.

Publisher's Note Springer Nature remains neutral with regard to jurisdictional claims in published maps and institutional affiliations. 